\title{
Adaptive NN Control for Multisteering Plane Aircraft with Dead Zone or Backlash Input Nonlinearity
}

\author{
Xiang-fei Meng, Ying Wang, and Mao-long Lv \\ Equipment Management and Safety Engineering College, Air Force Engineering University, Xi'an 710051, China \\ Correspondence should be addressed to Mao-long Lv; 18037707161@163.com
}

Received 17 March 2017; Accepted 30 May 2017; Published 22 June 2017

Academic Editor: Jean Jacques Loiseau

Copyright (C) 2017 Xiang-fei Meng et al. This is an open access article distributed under the Creative Commons Attribution License, which permits unrestricted use, distribution, and reproduction in any medium, provided the original work is properly cited.

\begin{abstract}
Considering that many factors such as actuator input dead zone, backlash, and external disturbance could affect the exactness of trajectory tracking, therewith a robust adaptive neural network control scheme on the basis of control allocation is proposed for the sake of tracking control of multisteering plane aircraft with actuator input dead zone or backlash nonlinearity. First of all, an actuator input dead zone or backlash nonlinearity control assignment model is established and the control allocation equation is derived. Secondly, the system nonlinear uncertainty is compensated by means of radial basis function neural network, and a robust term is introduced to achieve robustness against external disturbance and system errors. Finally, by utilizing Lyapunov stability theorem, it has been proved that all the signals in the closed-loop system are bounded, and the tracking error converges to a small residual set asymptotically. Simulation results on ICE101 multisteering plane aircraft demonstrate the outstanding tracking performance and strong robustness as well as effectiveness of the proposed approach, which can effectively overcome the adverse influence of dead zone, backlash nonlinearity, and external disturbance on the system.
\end{abstract}

\section{Introduction}

In order to improve the performance of aircraft, the multisteering surface configuration is used in the flight control design of advanced aircrafts $[1,2]$. The redundant actuators can not only improve the survivability of the multisteering plane aircraft in harsh environment [3] but also offer the control redundancy to avoid having the disadvantage of position saturation and rate saturation on the control surface, which grants the aircraft a better control performance. However, it also brings the difficulty in coping with the nonmatching uncertainty during designing controller. To solve the control design problem on uncertain interconnected nonlinear systems without the matching condition, many remarkable achievements have been made; for example, an adaptive fuzzy decentralized output-feedback control design is presented for a class of interconnected nonlinear purefeedback systems, the considered nonlinear systems contain unknown nonlinear uncertainties, and it is not necessary to measure the states directly [4]. In [5], an adaptive fuzzy robust output-feedback control problem is considered for a class of single-input and single-output nonlinear strictfeedback system with unstructured uncertainties, unknown dead zone, and the dynamics uncertainties. Moreover, fuzzy logic systems are also applied to identify the unknown nonlinear functions, and a state filter observer is designed to estimate the unmeasured states in $[6,7]$. In recent years, multisteering surface control technology for aircraft with dead zone or backlash input nonlinearity has been gradually developed $[8,9]$ and draws more and more attention from the aviation academics [10-13].

So far, the research on multisteering plane aircraft control distribution method has obtained a series of achievements, which mainly contain generalized inverse method [14], direct distribution method [15], and serial chain method [16]. These methods have achieved a good manipulation of the distribution of the effect under the condition that rudder command deflection angle is equal to the actual deflection angle. However, the dynamic characteristics of the actuator and nonlinear factors have been ignored [17]. In [18], the actuator is modeled as a first-order dynamic system, and the model predictive control theory is used to realize the dynamic 


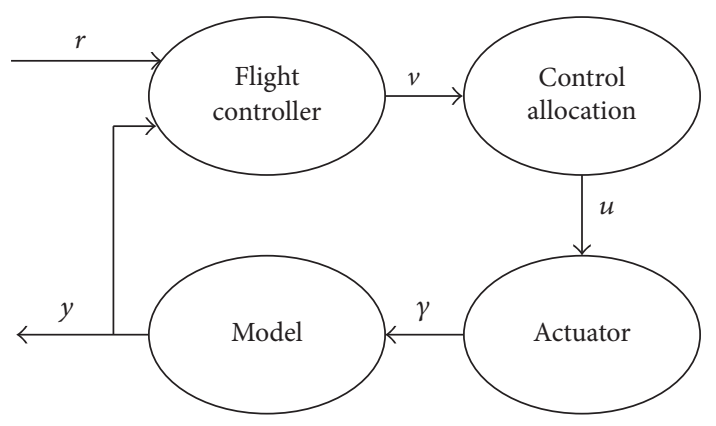

FIGURE 1: Structure of cascade connected flight control system.

control assignment of multimanipulated surface. In order to design the flight control command for aircraft with actuator input nonlinearity, [19] proposed an output-feedback control method with prescribed performance for single-input and single-output (SISO) switched non-strict-feedback nonlinear systems. Further, [20] proposed an adaptive fuzzy backstepping output-feedback tracking control approach for a class of multi-input and multi-output (MIMO) stochastic nonlinear systems. In addition, the actuators of multisteering plane aircraft show more nonlinear characteristics such as saturation, dead zone, backlash, and hysteresis, which will inevitably weaken the stability of the closed-loop system and even make the system unstable [21-23]. Therefore, it is of great practical significance and practical value to design the flight control command for multisteering plane aircraft in the case of nonlinearity, such as dead zone or backlash input nonlinearity.

In this note, a robust adaptive neural network control method is proposed for the multisteering plane aircraft tracking control problem with actuator input dead zone or backlash input nonlinearity. Based on the input nonlinear model with dead zone or backlash characteristic, the virtual control instruction is designed according to the control distribution equation. Then the radial basis neural network is introduced to compensate the system nonlinear uncertainty, and a robust term is employed to achieve robustness against external disturbance and system error. What is more, it has been rigorously proved that all signals of the closed-loop system are bounded, and the tracking error converges to a small residual set asymptotically using Lyapunov stability theorem. Finally, the simulation results on ICE101 multisteering plane aircraft demonstrate the effectiveness and excellent tracking performance of the proposed approach.

\section{Actuator Input Dead Zone or Backlash Nonlinear Control Assignment Modeling}

2.1. Control Assignment Modeling. The structure of the multisteering plane aircraft cascade flight control system shown in Figure 1 is different from the conventional flight control system. The control assignment module is introduced in Figure 1 to implement the assignment of the control surface. It is easy to know that the design of the multimaneuvering plane flight control law has been mainly divided into two steps: first, designing the virtual control instruction in the outer ring; secondly, designing the control dispenser and then controlling the multisteering plane aircraft by the actuator.

In [24], the kinetic model linearization is described as follows:

$$
\dot{\mathbf{x}}=\mathbf{M x}+\mathbf{f}_{\Delta}(\mathbf{x})+\mathbf{Z}_{\gamma} \boldsymbol{\gamma}+\mathbf{Z}_{\gamma} \mathbf{d}(\mathbf{x}, \boldsymbol{\gamma}, t),
$$

where $\mathbf{M} \in \mathbf{R}^{n_{x} \times n_{x}}$ is the system matrix; the continuous function $\mathbf{f}_{\Delta}(\mathbf{x})$ represents the unknown nonlinear uncertainties generated in the linearization process; $\mathbf{Z}_{\gamma} \in \mathbf{R}^{n_{x} \times m}$ is the control input matrix; $\mathbf{d}(\mathbf{x}, \boldsymbol{\gamma}, t)$ is the external disturbance or linearization error satisfying the matching condition.

Assumption 1. $\left(\mathbf{M}, \mathbf{Z}_{\gamma}\right)$ is controllable and there exists an unknown positive constant $d^{*}$ such that $\|\mathbf{d}(\mathbf{x}, \gamma, t)\| \leq d^{*}$.

The three-axis deflection angle acceleration has usually been chosen as the virtual control instruction to allocate the surface instructions; the corresponding control allocation equations are as follows:

$$
\begin{gathered}
\mathbf{v}=\mathbf{Z} \mathbf{u}, \\
\mathbf{Z}_{\gamma}=\mathbf{Z}_{v} \mathbf{Z},
\end{gathered}
$$

where $\mathbf{v} \in \mathbf{R}^{n}$ is the virtual instruction vector; $\mathbf{Z} \in \mathbf{R}^{n \times m}$ is control efficiency matrix, satisfying $\operatorname{rank}(\mathbf{Z})=n<m ; \mathbf{Z}_{v} \in$ $\mathbf{R}^{n_{x} \times n}$ is an enter matrix for the virtual controller; $\mathbf{u} \in \mathbf{R}^{m}$ is the rudder command deflection angle.

2.2. Actuator Nonlinearity Modeling. It is often assumed that the deflection angle of the rudder command is equal to the actual deflection angle of the rudder surface in the flight control design of multisteering plane aircraft [17], namely,

$$
\gamma=\mathbf{u}
$$

Thus, we can obtain

$$
\dot{\mathbf{x}}=\mathbf{M x}+\mathbf{Z}_{v} \mathbf{v}+\mathbf{f}_{\Delta}(\mathbf{x})+\mathbf{Z}_{\gamma} \mathbf{d}(\mathbf{x}, \boldsymbol{\gamma}, t) .
$$

In the steering gear, the actuator nonlinearity cannot be ignored due to the presence of dead zone, backlash, hysteresis, and so on, and that means $\gamma \neq \mathbf{u}$.

The nonlinear transmission model of the actuator is shown as follows:

$$
\gamma=\varphi \mathbf{u}+\boldsymbol{\varepsilon}_{\gamma}(\mathbf{u}),
$$

where $\boldsymbol{\varphi}=\operatorname{diag}\left\{\varphi_{1}, \varphi_{2}, \ldots, \varphi_{m}\right\}, \varphi_{i}>0$ is a known constant; $\boldsymbol{\varepsilon}_{\gamma}(\mathbf{u})=\left[\varepsilon_{\gamma 1}(\mathbf{u}), \varepsilon_{\gamma 2}(\mathbf{u}), \ldots, \varepsilon_{\gamma m}(\mathbf{u})\right]^{T}$ is unknown modeling error; and $\boldsymbol{\varepsilon}_{\gamma}(\mathbf{u}) \in \mathbf{R}^{m}$. It is easy to see that actuator linear model (4) is a special case of nonlinear model (6).

Control Object. Consider the actuator with nonlinear characteristics of (6); design a virtual control instruction $\mathbf{v}$ so that the system output $\mathbf{y}$ can track the desired state $\mathbf{r}$, where $\mathbf{y}=\mathbf{H x}, \mathbf{H} \in \mathbf{R}^{n \times n_{x}}$ is the system output matrix.

Assumption 2. The unknown modeling error $\boldsymbol{\varepsilon}_{y}(\mathbf{u})$ is bounded and there exists an unknown constant $\varepsilon_{\gamma}^{*}>0$ such that $\left\|\boldsymbol{\varepsilon}_{\gamma}(\mathbf{u})\right\| \leq \varepsilon_{\gamma}^{*}$. 
Model (6) is equivalent to $\gamma_{i}=\varphi_{i} u_{i}+\varepsilon_{\gamma i}(\mathbf{u})$ and it represents dead zone and backlash nonlinearity.

(i) Dead Zone Nonlinearity. When the actuator input nonlinearity is dead zone, its mathematical model can be described as follows:

$$
\gamma_{i}= \begin{cases}\varphi_{i}\left(u_{i}-z_{i, r}\right), & u_{i} \geq z_{i, r}, \\ 0, & -z_{i, l}<u_{i}<z_{i, r} \\ \varphi_{i}\left(u_{i}+z_{i, l}\right), & u_{i} \leq-z_{i, l},\end{cases}
$$

where $\varphi_{i}$ is the dead zone slope; $z_{i, r}>0$ and $z_{i, l}>0$ are the starting point and the end point of dead zone. Let

$$
\varepsilon_{\gamma i}(\mathbf{u})= \begin{cases}-\varphi_{i} z_{i, r}, & u_{i} \geq z_{i, r}, \\ -\varphi_{i} u_{i}, & -z_{i, l}<u_{i}<z_{i, r}, \\ \varphi_{i} z_{i, l}, & u_{i} \leq-z_{i, l} .\end{cases}
$$

The dead zone model (8) is consistent with model (7), since

$$
\left\|\boldsymbol{\varepsilon}_{\gamma}(\mathbf{u})\right\| \leq \sqrt{\left(\sum_{i=1}^{m}\left(\max \left\{\varphi_{i}\right\} \cdot \max \left\{\left|z_{i, r}\right|,\left|z_{i, l}\right|\right\}\right)^{2}\right)} .
$$

From (9), we can know the modeling error is bounded, indicating that the dead zone nonlinearity can be expressed by model (6) and conformed to Assumption 2.

(ii) Backlash Nonlinearity. When the actuator input nonlinearity is backlash, its mathematical model can be described as follows:

$$
\gamma_{i}= \begin{cases}\varphi_{i}\left(u_{i}-Z_{i, r}\right), & \dot{u}_{i}>0, \gamma_{i}=\varphi_{i}\left(u_{i}-Z_{i, r}\right) \\ \varphi_{i}\left(u_{i}-Z_{i, l}\right), & \dot{u}_{i}<0, \gamma_{i}=\varphi_{i}\left(u_{i}-Z_{i, l}\right) \\ \gamma_{i}\left(t_{-}\right), & \text {others, }\end{cases}
$$

where $\varphi_{i}>0$ is the backlash slope; $Z_{i, r}>0$ and $Z_{i, l}<0$ are the relevant position. Let

$$
\varepsilon_{\gamma i}(\mathbf{u})= \begin{cases}-\varphi_{i} Z_{i, r}, & \dot{u}_{i}>0, \gamma_{i}=\varphi_{i}\left(u_{i}-Z_{i, r}\right), \\ -\varphi_{i} Z_{i, l}, & \dot{u}_{i}<0, \gamma_{i}=\varphi_{i}\left(u_{i}-Z_{i, l}\right), \\ \gamma_{i}\left(t_{-}\right)-\varphi_{i} u_{i}, & \text { others. }\end{cases}
$$

Backlash model (11) is also consistent with model (6). Similarly, we can obtain

$$
\left\|\boldsymbol{\varepsilon}_{\gamma}(\mathbf{u})\right\| \leq\left(\sum_{i=1}^{m}\left(\varphi_{i} \cdot \max \left\{\left|Z_{i, r}\right|,\left|Z_{i, l}\right|\right\}\right)^{2}\right)^{1 / 2} .
$$

It is easy to know that error $\boldsymbol{\varepsilon}_{\gamma}(\mathbf{u})$ is bounded, which indicates that the backlash nonlinearity can be represented by model (6) and conformed to Assumption 2.
2.3. The Control Assignment Equation with Actuator Dead Zone or Backlash Nonlinearity. In order to allocate the virtual control instruction expected from the aircraft to each control surface, in the project, it is often solved as follows:

$$
\begin{array}{ll}
\min & \|\mathbf{B u}\| \\
\text { s.t. } & \mathbf{Z u}=\mathbf{v},
\end{array}
$$

where $\mathbf{B} \in \mathbf{R}^{m \times m}$ is a diagonal positive definite weight matrix, and the amount of steering surface can be adjusted to balance surface's use efficiency by changing $\mathbf{B}$.

The optimal control law of model (13) can be solved by the least squares method as follows:

$$
\mathbf{u}=\mathbf{B}^{-2} \mathbf{Z}^{T}\left(\mathbf{Z B}^{-2} \mathbf{Z}^{T}\right)^{-1} \mathbf{v} .
$$

Therefore, we have

$$
\begin{aligned}
\dot{\mathbf{x}}= & \mathbf{M} \mathbf{x}+\mathbf{Z}_{v}\left(\mathbf{Z} \boldsymbol{\varphi} \mathbf{B}^{-2} \mathbf{Z}^{T}\left(\mathbf{Z B}^{-2} \mathbf{Z}^{T}\right)^{-1} \mathbf{v}+\mathbf{Z} \boldsymbol{\varepsilon}_{\gamma}\right) \\
& +\mathbf{f}_{\Delta}(\mathbf{x})+\mathbf{Z}_{v} \mathbf{Z} \mathbf{d}(\mathbf{x}, \boldsymbol{\gamma}, t) .
\end{aligned}
$$

\section{Adaptive NN Controller Design}

The controllability of the system matrix in model (15) with actuator nonlinearity is investigated. Let

$$
\begin{aligned}
& \mathbf{Z}_{0}=\mathbf{Z} \boldsymbol{\varphi} \mathbf{B}^{-2} \mathbf{Z}^{T}\left(\mathbf{Z B}^{-2} \mathbf{Z}^{T}\right)^{-1}, \\
& \mathbf{Z}_{1}=\mathbf{Z}_{v} \mathbf{Z}_{0} .
\end{aligned}
$$

Theorem 3. System matrixes $\mathbf{M}$ and $\mathbf{Z}_{1}$ in model (15) are stable and controllable.

Proof. By Assumption 1, system matrix $\left(\mathbf{M}, \mathbf{Z}_{\gamma}\right)$ of the multisteering plane aircraft is controllable. So

$$
\operatorname{rank}\left[\mathbf{Z}_{\gamma}\left|\mathbf{M Z}_{\gamma}\right| \mathbf{M}^{2} \mathbf{Z}_{\gamma}|\cdots| \mathbf{M}^{n_{x}-1} \mathbf{Z}_{\gamma}\right]=n_{x} .
$$

Let

$$
\begin{aligned}
& \mathbf{S}_{\gamma}=\left[\mathbf{Z}_{\gamma}\left|\mathbf{M} \mathbf{Z}_{\gamma}\right| \mathbf{M}^{2} \mathbf{Z}_{\gamma}|\cdots| \mathbf{M}^{n_{x}-1} \mathbf{Z}_{\gamma}\right] \\
& \mathbf{S}_{v}=\left[\mathbf{Z}_{v}\left|\mathbf{M Z}_{v}\right| \mathbf{M}^{2} \mathbf{Z}_{v}|\cdots| \mathbf{M}^{n_{x}-1} \mathbf{Z}_{v}\right] .
\end{aligned}
$$

Combining (3) and (17), we have

$$
n_{x}=\operatorname{rank} \mathbf{S}_{\gamma}=\operatorname{rank}\left(\mathbf{S}_{v} \mathbf{Z}\right) \leq \operatorname{rank} \mathbf{S}_{v} \leq n_{x} .
$$

So $\operatorname{rank} \mathbf{S}_{v}=n_{x}$. Let

$$
\mathbf{S}_{1}=\left[\mathbf{Z}_{1}\left|\mathbf{M Z}_{1}\right| \mathbf{M}^{2} \mathbf{Z}_{1}|\cdots| \mathbf{M}^{n_{x}-1} \mathbf{Z}_{1}\right] .
$$

We can obtain

$$
\operatorname{rank} \mathbf{S}_{1}=\operatorname{rank}\left(\mathbf{S}_{v} \mathbf{Z}_{0}\right)=\operatorname{rank} \mathbf{S}_{v}=n_{x} .
$$

So $\left(\mathbf{M}, \mathbf{Z}_{1}\right)$ is controllable. The theorem is proved. 
From Theorem 3, for any given positive $k_{1}$ and positive definite matrix $\mathbf{J}$, there exists a positive definite matrix $\mathbf{P}$ such as the Riccati equation:

$$
\mathbf{M}^{T} \mathbf{P}+\mathbf{P M}-k_{1} \mathbf{P Z} \mathbf{Z}_{1}^{T} \mathbf{P}+\mathbf{J}=\mathbf{0} .
$$

Therefore, $\mathbf{M}-k_{1} \mathbf{Z}_{1} \mathbf{Z}_{1}^{T} \mathbf{P}$ is a Hurwitz matrix.

Define reference state $\mathbf{x}_{r}$ as

$$
\begin{aligned}
\mathbf{x}_{r} & =\left(k_{1} \mathbf{Z}_{1} \mathbf{Z}_{1}^{T} \mathbf{P}-\mathbf{M}\right)^{-1} \mathbf{Z}_{1} \mathbf{G}_{0}^{-1} \mathbf{r}, \\
\mathbf{G}_{0} & =\mathbf{H}\left(k_{1} \mathbf{Z}_{1} \mathbf{Z}_{1}^{T} \mathbf{P}-\mathbf{M}\right)^{-1} \mathbf{Z}_{1},
\end{aligned}
$$

where matrix $\mathbf{G}_{0}$ is reversible; otherwise it will not be possible to design a controller making $y$ track the desired state.

From (23), we have

$$
\begin{gathered}
\mathbf{H} \mathbf{x}_{r}=\mathbf{r}, \\
k_{1} \mathbf{Z}_{1} \mathbf{Z}_{1}^{T} \mathbf{P} \mathbf{x}_{r}-\mathbf{M} \mathbf{x}_{r}=\mathbf{Z}_{1} \mathbf{G}_{0}^{-1} \mathbf{r} .
\end{gathered}
$$

Define $\mathbf{e}_{r}=\mathbf{x}-\mathbf{x}_{r}$, then $\dot{\mathbf{e}}_{r}=\dot{\mathbf{x}}=\mathbf{M x}+\mathbf{Z}_{1} \mathbf{v}+\mathbf{f}_{\Delta}(\mathbf{x})+\mathbf{Z}_{v} \Delta$, where $\boldsymbol{\Delta}=\mathbf{Z} \boldsymbol{\varepsilon}_{\gamma}+\mathbf{Z} \mathbf{d}(\mathbf{x}, \boldsymbol{\gamma}, t)$ is a disturbance item.

Assumption 4. There exists an ideal control instruction $\mathbf{v}^{*}$ that makes the error dynamic system stable; then we can obtain

$$
\mathbf{M x}+\mathbf{f}_{\Delta}(\mathbf{x})+\mathbf{Z}_{v} \Delta+\mathbf{Z}_{1} \mathbf{v}^{*}=\mathbf{M}_{c} \mathbf{e},
$$

where $\mathbf{M}_{c}$ is a Hurwitz matrix. Then (26) can be rewritten as

$$
\mathbf{M x}+\mathbf{f}_{\Delta}(\mathbf{x})+\mathbf{Z}_{v} \Delta+\mathbf{Z}_{1} \mathbf{v}^{*}-\mathbf{M}_{c} \mathbf{e}=\mathbf{0} .
$$

According to (27), the ideal virtual control instruction can be constructed as a linear term $\mathbf{v}_{L}^{*}$, a nonlinear term $\mathbf{v}_{N}^{*}$, and a robust term $\mathbf{v}_{\Delta}^{*}$ such that

$$
\mathbf{v}^{*}=\mathbf{v}_{L}^{*}-\mathbf{v}_{N}^{*}+\mathbf{v}_{\Delta}^{*} \text {. }
$$

Then we can arrive at

$$
\begin{array}{r}
\mathbf{M x}-\mathbf{M}_{c} \mathbf{e}+\mathbf{Z}_{1} \mathbf{v}_{L}^{*}=\mathbf{0}, \\
\mathbf{f}_{\Delta}(\mathbf{x})-\mathbf{Z}_{1} \mathbf{v}_{N}^{*}=\mathbf{0}, \\
\mathbf{Z}_{v} \Delta+\mathbf{Z}_{1} \mathbf{v}_{\Delta}^{*}=\mathbf{0} .
\end{array}
$$

So (27) holds. Likewise, the virtual control law can be designed as $\mathbf{v}=\mathbf{v}_{L}-\mathbf{v}_{N}+\mathbf{v}_{\Delta}$, where $\mathbf{v}_{L}=\mathbf{G}_{0}^{-1} \mathbf{r}-k_{1} \mathbf{Z}_{1}^{T} \mathbf{P} \mathbf{x}$, $\mathbf{v}_{\Delta}=-k_{2} \operatorname{sgn}\left(\boldsymbol{\eta}\left(\mathbf{e}_{r}\right)\right)$ and $\boldsymbol{\eta}\left(\mathbf{e}_{r}\right)=\mathbf{Z}_{1}^{T} \mathbf{P} \mathbf{e}_{r}$, where $k_{1}$ and $k_{2}$ are positive design parameters. We use RBF neural network to approximate $\mathbf{v}_{N}^{*}$. Let

$$
\mathbf{v}_{N}^{*}=\mathbf{W}^{* T} \boldsymbol{\psi}(\mathbf{x})+\boldsymbol{\varepsilon},
$$

where $\mathbf{W}^{*} \in \mathbf{R}^{l \times n}$ is the neural network weight matrix; $\psi(\mathbf{x}) \epsilon$ $\mathbf{R}^{l}$ is the radial basis function vector; the approximation error vector $\boldsymbol{\varepsilon}=\left[\varepsilon_{1}, \varepsilon_{2}, \ldots, \varepsilon_{n}\right]^{T}$ satisfies $\left|\varepsilon_{i}\right| \leq \varepsilon_{i}^{*}$ with $\varepsilon_{i}^{*}$ being an unknown constant. Let

$$
\mathbf{v}_{N}=\widehat{\mathbf{W}}^{T} \psi(\mathbf{x}),
$$

where $\widehat{\mathbf{W}} \in \mathbf{R}^{l \times n}$ is the estimated value of $\mathbf{W}^{*}$, and the adaptive updating law of the weight estimation matrix is $\dot{\widehat{\mathbf{W}}}=\boldsymbol{\Gamma} \boldsymbol{\psi}(\mathbf{x}) \boldsymbol{\eta}^{T}\left(\mathbf{e}_{r}\right)$, where the diagonal matrix $\boldsymbol{\Gamma} \in \mathbf{R}^{l \times l}$ is an adaptive gain matrix. The error matrix $\widetilde{\mathbf{W}}=\mathbf{W}^{*}-\widehat{\mathbf{W}}$.

\section{Stability Analysis}

Theorem 5. Considering that the actuator has a multisteering plane equation (1) with dead zone or backlash nonlinearity, if the virtual instruction control law (30) is used under Assumptions 1-4, we have

$$
\lim _{t \rightarrow \infty} \mathbf{e}_{r}(t)=\mathbf{0}
$$

And all signals of the closed-loop system are stable.

Proof. Noting $\mathbf{v}=\mathbf{v}_{L}-\mathbf{v}_{N}+\mathbf{v}_{\Delta}$ and $\mathbf{v}_{L}=\mathbf{G}_{0}^{-1} \mathbf{r}-k_{1} \mathbf{Z}_{1}^{T} \mathbf{P x}$, we have

$$
\begin{aligned}
\dot{\mathbf{e}}_{r}= & \mathbf{M x}+\mathbf{Z}_{1}\left(\mathbf{G}_{0}^{-1} \mathbf{r}-k_{1} \mathbf{Z}_{1}^{T} \mathbf{P} \mathbf{x}-\mathbf{v}_{N}+\mathbf{v}_{\Delta}\right)+\mathbf{f}_{\Delta}(\mathbf{x}) \\
& +\mathbf{Z}_{v} \Delta .
\end{aligned}
$$

According to (25), we can arrive at

$$
\begin{aligned}
\dot{\mathbf{e}}_{r}= & \mathbf{M e}_{r}-k_{1} \mathbf{Z}_{1} \mathbf{Z}_{1}^{T} \mathbf{P \mathbf { e } _ { r }}+\mathbf{Z}_{1}\left(-\mathbf{v}_{N}+\mathbf{v}_{\Delta}\right)+\mathbf{f}_{\Delta}(\mathbf{x}) \\
& +\mathbf{Z}_{v} \Delta .
\end{aligned}
$$

Considering the following Lyapunov function,

$$
V=\frac{1}{2} \mathbf{e}_{r}^{T} \mathbf{P} \mathbf{e}_{r}+\frac{1}{2} \operatorname{tr}\left\{\widetilde{\mathbf{W}}^{T} \boldsymbol{\Gamma}^{-1} \widetilde{\mathbf{W}}\right\} .
$$

The time derivative of $V$ is equal to

$$
\begin{aligned}
\dot{V}= & \frac{1}{2} \mathbf{e}_{r}^{T}\left(\mathbf{M}^{T} \mathbf{P}+\mathbf{P M}\right) \mathbf{e}_{r}-k_{1} \mathbf{e}_{r}^{T} \mathbf{P} \mathbf{Z}_{1} \mathbf{Z}_{1}^{T} \mathbf{P} \mathbf{e}_{r} \\
& +\boldsymbol{\eta}^{T}\left(\mathbf{e}_{r}\right)\left(-\mathbf{v}_{N}+\mathbf{v}_{\Delta}\right)+\mathbf{e}_{r}^{T} \mathbf{P}\left(\mathbf{f}_{\Delta}(\mathbf{x})+\mathbf{Z}_{v} \boldsymbol{\Delta}\right) \\
& -\operatorname{tr}\left\{\widetilde{\mathbf{W}}^{T} \boldsymbol{\Gamma}^{-1} \dot{\hat{\mathbf{W}}}\right\} .
\end{aligned}
$$

Since $\mathbf{e}_{r}^{T} \mathbf{P Z} \mathbf{Z}_{1} \mathbf{Z}_{1}^{T} \mathbf{P} \mathbf{e}_{r} \geq 0$, we can obtain

$$
\begin{aligned}
\dot{V} \leq & -\frac{1}{2} \mathbf{e}_{r}^{T} \mathbf{J} \mathbf{e}_{r}+\boldsymbol{\eta}^{T}\left(\mathbf{e}_{r}\right)\left(-\mathbf{v}_{N}+\mathbf{v}_{\Delta}\right)+\mathbf{e}_{r}^{T} \mathbf{P} \mathbf{f}_{\Delta}(\mathbf{x}) \\
& +\mathbf{e}_{r}^{T} \mathbf{P} \mathbf{Z}_{v} \Delta-\operatorname{tr}\left\{\widetilde{\mathbf{W}}^{T} \boldsymbol{\Gamma}^{-1} \dot{\widehat{\mathbf{W}}}\right\} .
\end{aligned}
$$

Noting $\mathbf{e}_{r}^{T} \mathbf{P} \mathbf{Z}_{v} \boldsymbol{\Delta}=\boldsymbol{\eta}^{T}\left(\mathbf{e}_{r}\right) \mathbf{Z}_{0}^{-1} \boldsymbol{\Delta}$ and $\boldsymbol{\eta}^{T}\left(\mathbf{e}_{r}\right) \mathbf{v}_{N}^{*}=\mathbf{e}_{r}^{T} \mathbf{P} \mathbf{Z}_{1} \mathbf{v}_{N}^{*}$, we can arrive at

$$
\begin{aligned}
\dot{V} \leq & -\frac{1}{2} \mathbf{e}_{r}^{T} \mathbf{J} \mathbf{e}_{r}+\boldsymbol{\eta}^{T}\left(\mathbf{e}_{r}\right)\left(\mathbf{v}_{N}^{*}-\mathbf{v}_{N}+\mathbf{v}_{\Delta}+\mathbf{Z}_{0}^{-1} \Delta\right) \\
& +\mathbf{e}_{r}^{T} \mathbf{P}\left(\mathbf{f}_{\Delta}(\mathbf{x})-\mathbf{Z}_{1} \mathbf{v}_{N}^{*}\right)-\operatorname{tr}\left\{\widetilde{\mathbf{W}}^{T} \boldsymbol{\Gamma}^{-1} \dot{\hat{\mathbf{W}}}\right\} .
\end{aligned}
$$

Since $\mathbf{v}_{\Delta}=-k_{2} \operatorname{sgn}\left(\boldsymbol{\eta}\left(\mathbf{e}_{r}\right)\right), \mathbf{v}_{N}^{*}=\mathbf{W}^{* T} \boldsymbol{\psi}(\mathbf{x})+\boldsymbol{\varepsilon}$, and $\mathbf{v}_{N}=$ $\widehat{\mathbf{W}}^{T} \psi(\mathbf{x})$, it yields

$$
\begin{aligned}
\dot{V} \leq & -\frac{1}{2} \mathbf{e}_{r}^{T} \mathbf{J} \mathbf{e}_{r}-\operatorname{tr}\left\{\widetilde{\mathbf{W}}^{T} \boldsymbol{\psi}(\mathbf{x}) \boldsymbol{\eta}^{T}\left(\mathbf{e}_{r}\right)\right\} \\
& +\boldsymbol{\eta}^{T}\left(\mathbf{e}_{r}\right)\left(\widetilde{\mathbf{W}}^{T} \boldsymbol{\psi}(\mathbf{x})+\boldsymbol{\varepsilon}\right) \\
& +\boldsymbol{\eta}^{T}\left(\mathbf{e}_{r}\right)\left(\mathbf{Z}_{0}^{-1} \boldsymbol{\Delta}-k_{2} \operatorname{sgn}\left(\boldsymbol{\eta}\left(\mathbf{e}_{r}\right)\right)\right) .
\end{aligned}
$$


Due to $\operatorname{tr}\left\{\widetilde{\mathbf{W}}^{T} \boldsymbol{\psi}(\mathbf{x}) \boldsymbol{\eta}^{T}\left(\mathbf{e}_{r}\right)\right\}=\boldsymbol{\eta}^{T}\left(\mathbf{e}_{r}\right) \widetilde{\mathbf{W}}^{T} \boldsymbol{\psi}(\mathbf{x})$, we have

$$
\dot{V} \leq-\frac{1}{2} \mathbf{e}_{r}^{T} \mathbf{J e}_{r}+\boldsymbol{\eta}^{T}\left(\mathbf{e}_{r}\right)\left(\boldsymbol{\varepsilon}-k_{2} \operatorname{sgn}\left(\boldsymbol{\eta}\left(\mathbf{e}_{r}\right)\right)+\mathbf{Z}_{0}^{-1} \boldsymbol{\Delta}\right) .
$$

Noting that $\mathbf{d}(\mathbf{x}, \boldsymbol{\gamma}, t), \boldsymbol{\varepsilon}_{\gamma}$, and $\boldsymbol{\varepsilon}$ are bounded, so $\left\|\mathbf{Z}_{0}^{-1} \Delta\right\|$ and $\|\boldsymbol{\varepsilon}\|$ are bounded. Let $\left\|\mathbf{Z}_{0}^{-1} \Delta\right\| \leq z_{0}$ and $\|\boldsymbol{\varepsilon}\| \leq \varepsilon^{*}$ with $z_{0}$ and $\varepsilon^{*}$ being unknown constants. Then

$$
\dot{V} \leq-\frac{1}{2} \mathbf{e}_{r}^{T} \mathbf{J} \mathbf{e}_{r}+\left(\varepsilon^{*}+z_{0}-k_{2}\right) \sum_{i=1}^{m}\left|\left(\mathbf{e}_{r}^{T} \mathbf{P} \mathbf{Z}_{1}\right)_{i}\right| .
$$

Choosing the design parameters $k_{2}$ such that $k_{2} \geq \varepsilon^{*}+z_{0}$, then

$$
\dot{V} \leq-\frac{1}{2} \mathbf{e}_{r}^{T} \mathbf{J} \mathbf{e}_{r}
$$

Using the Rayleigh inequality $-\mathbf{e}_{r}^{T} \mathbf{J} \mathbf{e}_{r} \leq-\lambda_{\min }(\mathbf{J})\left\|\mathbf{e}_{r}\right\|^{2}$, we can obtain

$$
\dot{V} \leq-\frac{1}{2} \lambda_{\min }(\mathbf{J})\left\|\mathbf{e}_{r}\right\|^{2}
$$

Then, we can further arrive at

$$
\lim _{t \rightarrow \infty} \lambda_{\min }(\mathbf{J}) \int_{t_{0}}^{t} \mathbf{e}_{r}^{T}(\tau) \mathbf{e}_{r}(\tau) d \tau \leq 2 V\left(t_{0}\right)
$$

According to Barbalat's lemma, we can know $\lim _{t \rightarrow \infty} \mathbf{e}_{r}(t)=0$, so $\|\widetilde{\mathbf{W}}\|$ is convergent, and all the signals are convergent. The theorem is proved.

From Theorem 5, we can know that the virtual control law $\mathbf{v}=\mathbf{v}_{L}-\mathbf{v}_{N}+\mathbf{v}_{\Delta}$ designed in this paper can be applied to the actuator dead zone or backlash nonlinearity and can effectively eliminate external disturbance and system error.

\section{Simulation Analysis}

In order to verify the effectiveness of the proposed method, the ICE101 tailless aircraft is taken as an example. The motion equation of the aircraft can be described as follows [25]:

$$
\dot{\mathbf{x}}=\mathbf{M x}+\mathbf{Z}_{\gamma} \boldsymbol{\gamma}+\mathbf{f}_{\Delta}(\mathbf{x})+\mathbf{Z}_{\gamma} \mathbf{d}(\mathbf{x}, \boldsymbol{\gamma}, t),
$$

where $\mathbf{x}=[\alpha, \beta, p, j, r]^{T}$ are attack angle, side slip angle, roll angular rate, pitch angle rate, and yaw rate, respectively. Select the left and right ailerons, pitching flaps and left and right fullwing five rudder surface $\gamma=\left[\gamma_{\mathrm{el}}, \gamma_{\mathrm{er}}, \gamma_{\mathrm{pflap}}, \gamma_{\mathrm{amtl}}, \gamma_{\mathrm{amtr}}\right]^{T}$, the control surface position constraints, and rate constraints as follows:

$$
\begin{aligned}
& {[-30,-30,-30,0,0]^{T} \leq 57.3 \cdot \gamma^{T}} \\
& \quad \leq[30,30,30,60,60]^{T}, \\
& -[150,150,50,150,150]^{T} \leq 57.3 \cdot \dot{\gamma}^{T} \\
& \quad \leq[150,150,50,150,150]^{T} .
\end{aligned}
$$

The system matrix is described as follows:

M

$$
\begin{aligned}
& =\left[\begin{array}{ccccc}
-0.6344 & 0.0027 & 0 & 0.9871 & 0 \\
0 & -0.0038 & 0.1540 & 0 & -0.9876 \\
0 & -8.2125 & -0.7849 & 0 & 0.1171 \\
-0.5971 & 0 & 0 & -0.5099 & 0 \\
0 & -0.8887 & -0.0299 & 0 & -0.0156
\end{array}\right], \\
& \mathbf{Z}_{\gamma} \\
& =\left[\begin{array}{ccccc}
-0.0459 & -0.0459 & -0.0395 & -0.0133 & -0.0133 \\
-0.0047 & 0.0047 & 0 & 0.0031 & -0.0031 \\
3.7830 & -3.7830 & 0 & 1.8255 & -1.8255 \\
-2.5114 & -2.5115 & -1.9042 & -0.9494 & -0.9494 \\
0.0453 & -0.0453 & 0 & -0.2081 & 0.2081
\end{array}\right] .
\end{aligned}
$$

In order to verify the validity about overcoming the adverse influence caused by actuator input nonlinearity and external disturbance of the method designed in this paper, similar to the literature [17], the reference instruction $\mathbf{r}=$ $[\alpha, \beta, p]^{T}$ can be set to apply a $5^{\circ}$ angle of attack command to the airplane during $t=7 \sim 13 \mathrm{~s}$. When $t=6 \sim 16 \mathrm{~s}$, the roll angle command is applied to the aircraft and the side slip angle command is always set to zero.

The Gaussian function is chosen as the basis function of the RBF neural network, whose parameters are set as follows: the number of nodes $l=64$ and the network center $\mu_{i}(i=$ $1,2, \ldots, 5)$ are evenly distributed in $[-2.5,4] \times[-2.5,4] \times$ $[-2.5,4] \times[-2.5,4] \times[-2.5,4]$; the initial value of the neural network weights are set to zero. The controller parameters $k_{1}=1.5, k_{2}=2.5$, and $v=0.25$, adaptive gain matrix $\Gamma=5 \mathbf{I}$, $\mathbf{B}=4 \mathbf{I}$, and $\mathbf{J}=\operatorname{diag}\{20,15,15,1,10\}$. Choose the dead zone model to be

$$
\gamma_{i}= \begin{cases}1.12\left(u_{i}-0.025\right), & u_{i} \geq 0.025, \\ 0, & -0.025<u_{i}<0.025 \\ 1.12\left(u_{i}+0.025\right), & u_{i} \leq-0.025\end{cases}
$$

and external disturbances and uncertainties are selected as follows:

$$
\begin{aligned}
& \mathbf{d}(\mathbf{x}, \gamma, t) \\
& =0.012 \cdot\left[0.35 \sin t, \cos \alpha, 0.1 \sin ^{2} t, \cos ^{2} t, 0\right]^{T}, \\
& \mathbf{f}_{\Delta}(\mathbf{x}) \\
& =-0.12\left[0, \sin ^{2} \beta, \sin \alpha \cos p, \cos ^{2} j, 0.01 \sin ^{2}(j r)\right]^{T} .
\end{aligned}
$$



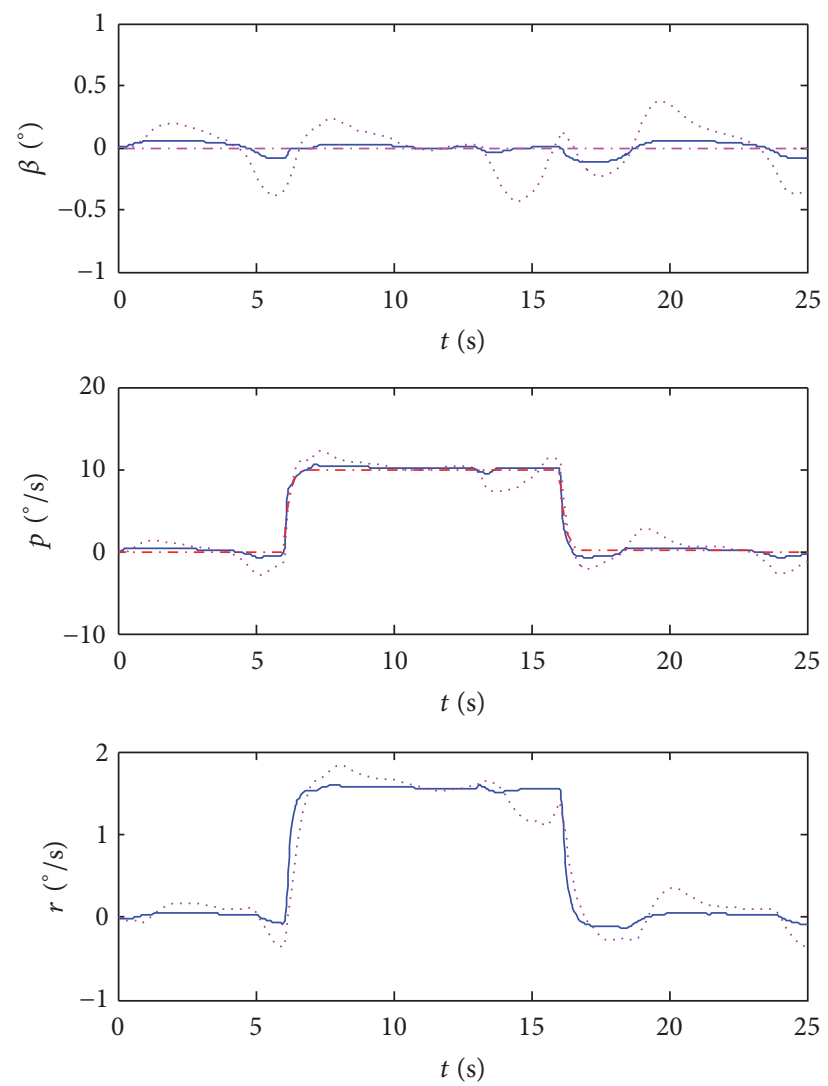

Figure 2: State curves with dead zone.

(i) Actuator Input Dead Zone Nonlinearity. Simulate the virtual control law $\mathbf{v}$ with uncertain term, interference term, and dead zone nonlinearity. The obtained flight state and the deflection curves of aircrafts are shown in Figures 2 and 3.

According to Figure 2, it can be seen that the proposed virtual control law can ensure that the angle of attack $\alpha$, side angle $\beta$, and roll angle rate $p$ can effectively overcome the adverse influences leading to actuator input dead zone input nonlinearity and external disturbance, while the conventional method in [17] has a bigger tracking error in tracking the reference command signal.

In Figure 3, when the reference command is zero, the left side of the whole wing cannot return to the equilibrium position, which is not conducive to steady flight. It is easy to see that the tracking effect of our scheme is superior to that of [17] when considering actuator input dead zone nonlinearity.

(ii) Actuator Input Backlash Nonlinearity. To further verify the effectiveness of the proposed method, the actuator input backlash nonlinearity is considered for simulation. Keep all simulation parameters unchanged; set the backlash nonlinearity parameters as $\varphi_{i}=1.25, Z_{i, r}=1 / 57.3, Z_{i, l}=-1 / 57.3$, $i=1,2, \ldots, 5$.

Figures 4 and 5 are the corresponding flight state and control surface deflection dynamic curves, respectively. In Figure 4 , it can be seen that the proposed method can
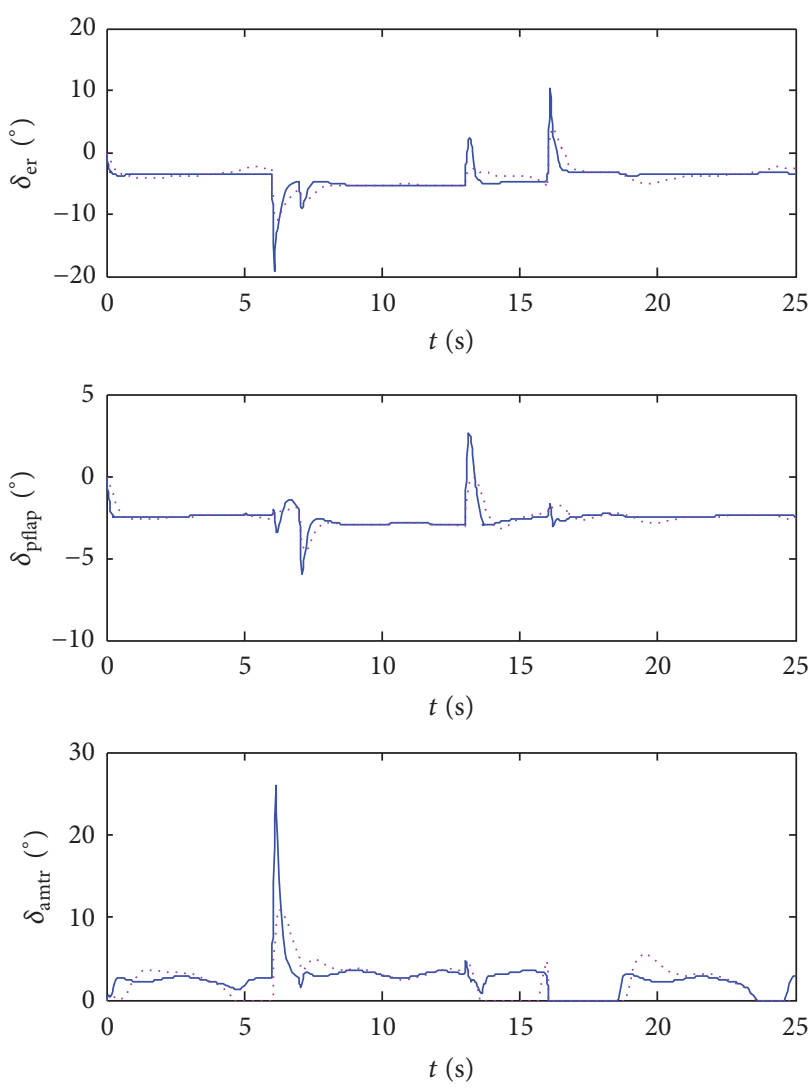

Figure 3: Dynamic curves with dead zone.
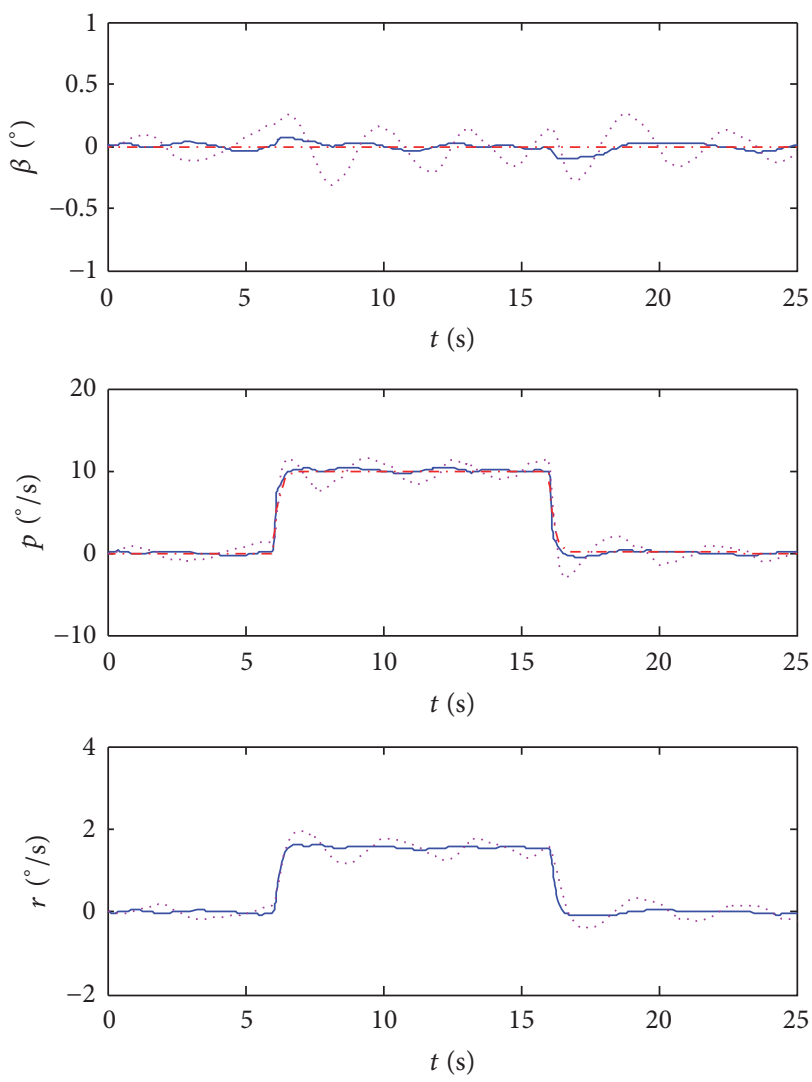

FIGURE 4: State curves with backlash. 

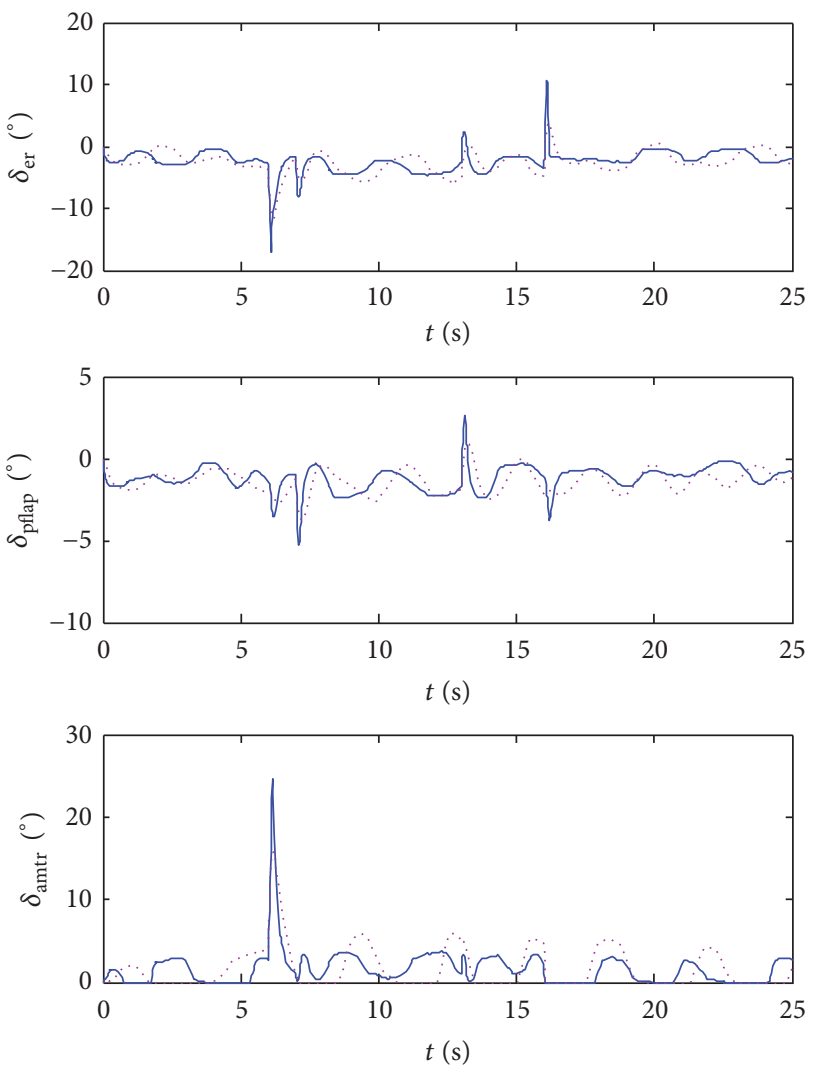

FIGURe 5: Dynamic curves with backlash.

ensure that the angle of attack $\alpha$, side angle $\beta$, and roll angle rate $p$ can effectively overcome the adverse influences caused by actuator input backlash nonlinearity and external disturbance. When the actuator is coupled with the backlash nonlinearity, it follows that, from Figure 5, we can know that the proposed method can obtain almost the same good tracking control performance as dead zone nonlinearity, and the tracking performance is superior to that of [17].

\section{Conclusion}

In this paper, a robust adaptive neural tracking control method is presented based on control assignment equation. The main advantages are as follows: firstly, the neural network is used to eliminate adverse effect of nonmatching uncertainty in the aircraft equations; secondly, the virtual control law designed in this paper can not only be applied to the actuator input dead zone but also be suitable for backlash input nonlinearity and can eliminate external disturbance and system error effectively; thirdly, Lyapunov stability theory is utilized to prove the stability of the closed-loop system, and the tracking error converges to a small residual set asymptotically; finally, the simulation on ICE101 multisteering plane aircraft shows that the proposed scheme has a better tracking performance and robustness than the approach of [17].

\section{Conflicts of Interest}

The authors declare that there are no conflicts of interest regarding the publication of this paper.

\section{Acknowledgments}

This work is supported by the National Natural Science Foundation of China (Grant no. 71601183).

\section{References}

[1] L. Wang, L. X. Wang, and C. R. Jia, "Methods on the control assignment of multi-steeringled flying wing layout combat aircraft," Acta Aerospace Sinica, vol. 32, no. 4, pp. 571-579, 2011.

[2] W. L. Jiang and C. Y. Dong, "Fault tolerant control of a class of variant aircraft based on control assignment," Journal of Beijing University of Aeronautics and Astronautics, vol. 40, no. 3, pp. 355-359, 2014 (Chinese).

[3] Y. Chen, X. M. Dong, and J. P. Xue, "Multi-objective optimization design of weight coefficient for weighted control assignment strategy," Control and Decision, vol. 28, no. 7, pp. 991-1001, 2013 (Chinese).

[4] Y. M. Li, S. C. Tong, and T. S. Li, "Adaptive fuzzy output feedback dynamic surface control of interconnected nonlinear pure-feedback systems," IEEE Transactions on Cybernetics, vol. 45, no. 1, pp. 138-149, 2015.

[5] Y. Li, S. Tong, Y. Liu, and T. Li, "Adaptive fuzzy robust output feedback control of nonlinear systems with unknown dead zones based on a small-gain approach," IEEE Transactions on Fuzzy Systems, vol. 22, no. 1, pp. 164-176, 2014.

[6] M.-1. Lv, X.-x. Sun, and S.-g. Liu, "An adaptive dynamic surface controller for ultra-low altitude airdrop flight path angle with actuator input nonlinearity," Mathematical Problems in Engineering, vol. 3, no. 1, pp. 1-9, 2016.

[7] Y. M. Li and S. C. Tong, "Adaptive fuzzy output-feedback control of pure-feedback uncertain nonlinear systems with unknown dead-zone," IEEE Transactions on Fuzzy Systems, vol. 22, no. 5, pp. 1341-1347, 2014.

[8] B. Jiang, Q. Shen, and P. Shi, "Neural-networked adaptive tracking control for switched nonlinear pure-feedback systems under arbitrary switching," Automatica. A Journal of IFAC, the International Federation of Automatic Control, vol. 61, pp. 119125, 2015.

[9] X. Zhao, X. Zheng, B. Niu, and L. Liu, "Adaptive tracking control for a class of uncertain switched nonlinear systems," Automatica, vol. 52, pp. 185-191, 2015.

[10] S. Tong, Y. Li, and P. Shi, "Observer-based adaptive fuzzy backstepping output feedback control of uncertain MIMO pure-feedback nonlinear systems," IEEE Transactions on Fuzzy Systems, vol. 20, no. 4, pp. 771-785, 2012.

[11] S. Tong and Y. Li, "Adaptive fuzzy output feedback tracking backstepping control of strict-feedback nonlinear systems with unknown dead zones," IEEE Transactions on Fuzzy Systems, vol. 20, no. 1, pp. 168-180, 2012.

[12] L. Long and J. Zhao, "Decentralized adaptive neural outputfeedback DSC for switched large-scale nonlinear systems," in IEEE Transactions on Cybernetics, 2016.

[13] W. Meng, Q. Yang, S. Jagannathan, and Y. Sun, "Adaptive neural control of high-order uncertain nonaffine systems: a transformation to affine systems approach," Automatica, vol. 50, no. 5, pp. 1473-1480, 2014. 
[14] D. Raney, R. Montgomery, and L. Green, "Flight control using distributed shape-change effector arrays," in Proceedings of the 41st Structures, Structural Dynamics, and Materials Conference and Exhibit, Atlanta, GA, USA, 2000.

[15] W. C. Durham, "Constrained control allocation," Journal of Guidance, Control, and Dynamics, vol. 16, no. 4, pp. 717-725, 1993.

[16] J. M. Buffington and D. F. Enns, "Lyapunov stability analysis of daisy chain control allocation," Journal of Guidance, Control, and Dynamics, vol. 19, no. 6, pp. 1226-1230, 1996.

[17] O. Harkegard and G. S. Torkel, "Resolving actuator redundancy optimal control vs. control allocation," Automatica, vol. 41, no. 1, pp. 137-144, 2005.

[18] Y. Luo, A. Serrani, S. Yurkovich, M. W. Oppenheimer, and D. B. Doman, "Model-predictive dynamic control allocation scheme for reentry vehicles," Journal of Guidance, Control, and Dynamics, vol. 30, no. 1, pp. 100-113, 2007.

[19] Y. Li, S. Tong, L. Liu, and G. Feng, "Adaptive output-feedback control design with prescribed performance for switched nonlinear systems," Automatica, vol. 80, pp. 225-231, 2017.

[20] Y. Li, S. Tong, and T. Li, “Observer-based adaptive fuzzy tracking control of MIMO stochastic nonlinear systems with unknown control direction and unknown dead-zones," IEEE Transactions on Fuzzy Systems, vol. 23, no. 4, pp. 1228-1241, 2014.

[21] H. Li, Y. A. Zhang, and F. Sun, "Multi-linear adaptive dynamic control allocation for multi-steering plane aircraft global set," Control and Decision, vol. 28, no. 3, pp. 379-390, 2013 (Chinese).

[22] C. K. Ahn, L. Wu, and P. Shi, "Stochastic stability analysis for 2D Roesser systems with multiplicative noise," Automatica, vol. 69, pp. 356-363, 2016.

[23] T. Achilles and G. A. Rovithakis, "A simplified adaptive neural network prescribed performance controller for uncertain MIMO feedback linearizable systems," IEEE Transactions on Neural Networks and Learning Systems, vol. 26, no. 3, pp. 589600, 2015.

[24] W. MacKunis, P. M. Patre, M. K. Kaiser, and W. E. Dixon, "Asymptotic tracking for aircraft via robust and adaptive dynamic inversion methods," IEEE Transactions on Control Systems Technology, vol. 18, no. 6, pp. 1448-1456, 2010.

[25] J. M. Buffington, "Tailless aircraft control allocation," in Proceedings of the Guidance, Navigation, and Control Conference, pp. 737-747, 1997. 


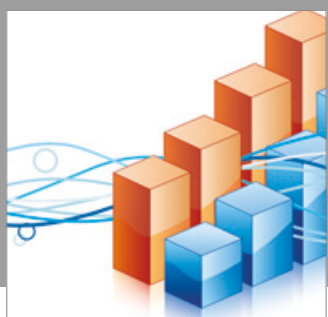

Advances in

Operations Research

vatersals

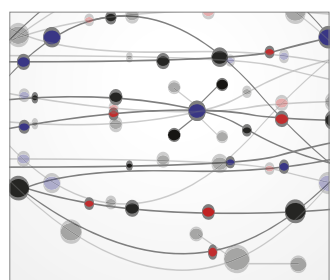

\section{The Scientific} World Journal
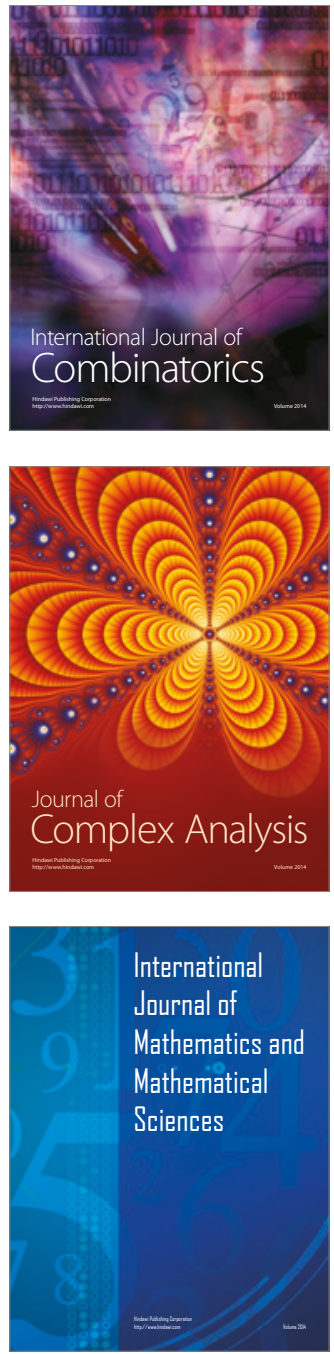
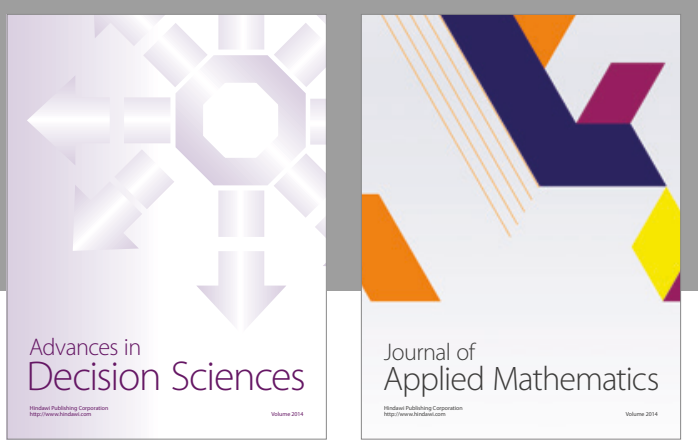

Algebra

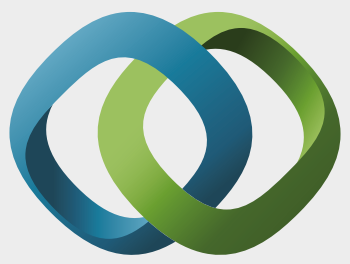

\section{Hindawi}

Submit your manuscripts at

https://www.hindawi.com
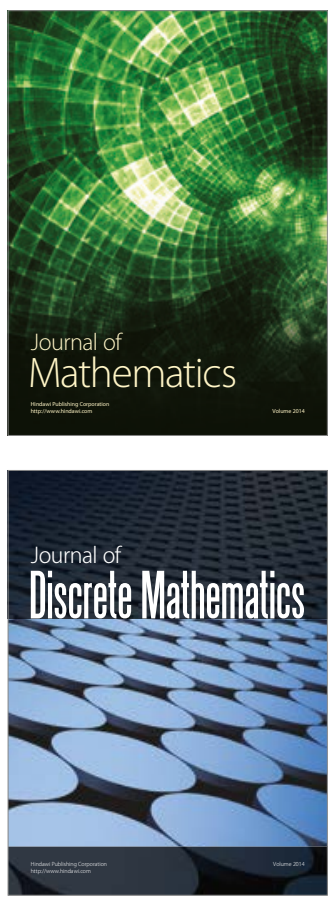

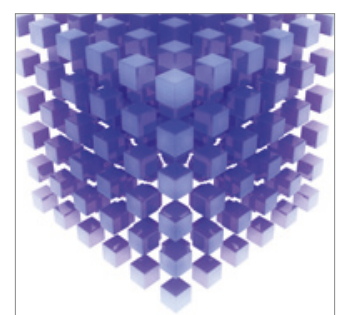

Mathematical Problems in Engineering
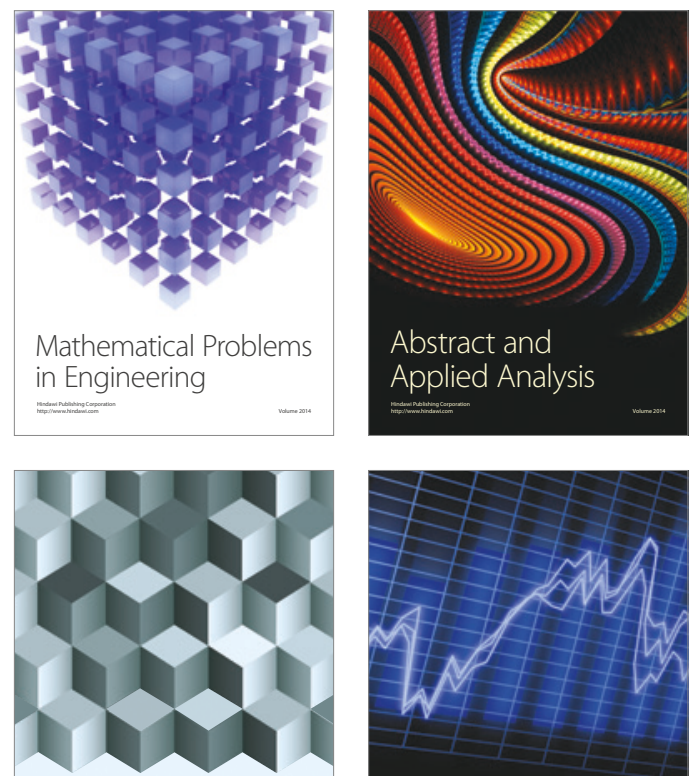

Journal of

Function Spaces

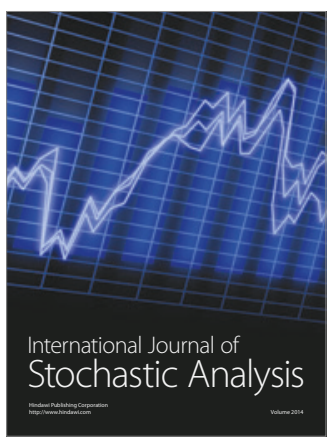

Probability and Statistics
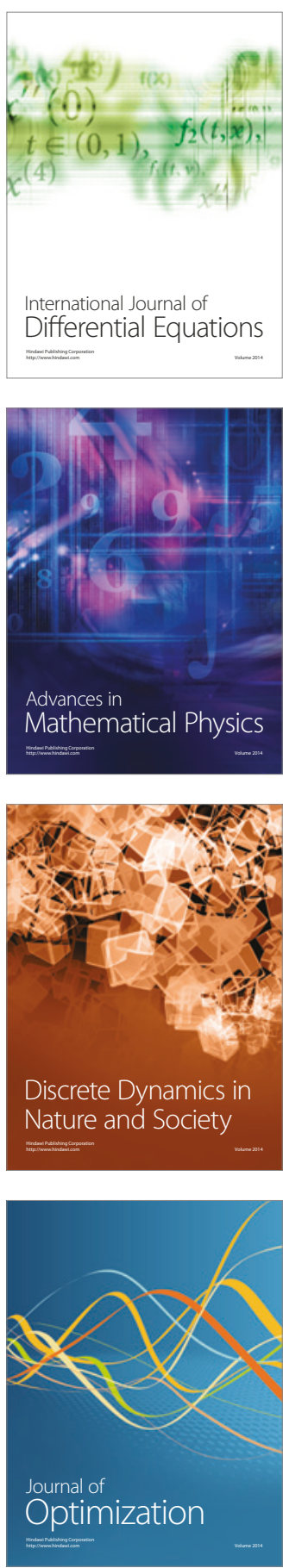